\section{Erste Erfahrungen mit Buprenorphin in der Methadonschwerpunktpraxis Mannheim}

Inge Hönekopp

Methadonschwerpunktpraxis, Mannheim
Zusammenfassung: Nach der Zulassung von Buprenorphin zur Substitutionsbehandlung in der Bundesrepublik werden nun erste Erfahrungen gesammelt. Anhand eines Falles werden sowohl mögliche Indikationen als auch Probleme bei der Umstellung von Methadon auf Buprenorphin dargestellt. Indikation zur Umstellung war die psychiatrische Komorbidität, in welcher auch eine Ursache für den unbefriedigenden Verlauf unter der Methadonsubstitutionsbehandlung gesehen wurde. Es wird gezeigt, dass bei unveränderter Fortführung des therapeutischen Settings durch Einsatz eines anderen Substitutes mit anderer Pharmakokinetik eine Verbesserung im Verlauf bewirkt werden kann.

Schlüsselwörter: Opiatabhängigkeit - Substitutionsbehandlung - Buprenorphin - Komorbidität - Compliance

First Experiences with Buprenorphine: After the recent authorisation of buprenorphine to the maintenance therapy of opiat addiction in Germany first experiences are done. We present a case which shows possible indications and problems during the change of methadone to buprenorphine. Indication for the change of medication was the psychiatric comorbidity as one probable cause for the non-satisfactory results of the ongoing methadone therapy. Without modification of the existing psychotherapeutic setting the change to a substitute with different pharmacocinetic properties ameliorated the outcoming clinical results.

Key words: Opiate Addiction - Maintenance Therapy - Buprenorphine - Comorbidity - Compliance

\section{Darstellung anhand eines Beispiels}

Zunächst: Wir haben lange auf die Zulassung von Buprenorphin (Subutex ${ }^{\circledast}$ ) zur Substitution in der Bundesrepublik gewartet. Im Vorfeld haben wir uns schon Gedanken gemacht, für welche PatientInnen wir eine Behandlung mit Buprenorphin an Stelle von Methadon für indiziert halten.

\section{Unsere Indikationen}

Als die Zulassung erfolgte, erstellten wir analog zu unserem Methadonaufklärungsbogen ein Patientenmerkblatt, welches wir im Wartezimmer auslegten und regen Absatz fand. Bei Neueinstellungen besprechen wir ohnehin alle Therapiemöglichkeiten und somit auch die verschiedenen zur Verfügung stehenden Medikamente.

Die Entscheidung zum „Mittel der Wahl“ wird gemeinsam mit dem Patienten / der Patientin getroffen.

Wir erwähnen, dass bei wesentlich mehr PatientInnen o.e. Indikationen bestehen, auch Interesse an Buprenorphin von Seiten der PatientInnen geäußert wird, jedoch die fehlende Möglichkeit, sich take-home „erarbeiten“ zu können, der Grund für den Verzicht auf eine Buprenorphin-Behandlung darstellt. Seit Februar behandelten/behandeln wir $12 \mathrm{~Pa}-$ tientInnen mit Buprenorphin. Gelegentlich sprachen wir auch bereits Substituierte, bei welchen wir eine Indikation sahen, auf einen Wechsel des Medikamentes an. So auch bei der folgenden Patientin, welche wir vorstellen möchten.

Frau A., 43 Jahre alt, befand sich seit dem 23.6 .98 bei einem Kollegen in Methadonsubstitution und wurde am 4.1.99 von

Tab. 1 Unsere Indikationen (Anzahl > 12, da Mehrfachnennungen)

\begin{tabular}{|c|c|c|}
\hline Indikation & Begründung & Anzahl \\
\hline Schwangerschaft & geringeres NAS & 2 \\
\hline $\begin{array}{l}\text { Kurzzeitabhängige, } \\
\text { Monotoxikomanie }\end{array}$ & $\begin{array}{l}\text { geringeres Abhängigkeits- } \\
\text { potenzial/unsere Erfahrung, } \\
\text { dass unter Methadon- } \\
\text { Substitution aus Monotoxi- } \\
\text { komanen häufig } \\
\text { Politoxikomane wurden! }\end{array}$ & 1 \\
\hline Überbrückungssubstitution & leichtere Entgiftung & 2 \\
\hline $\begin{array}{l}\text { ambulante Entgiftung, } \\
\text { auch von Methadon }\end{array}$ & leichtere Entgiftung & 3 \\
\hline $\begin{array}{l}\text { psychiatrische } \\
\text { Komorbidität }\end{array}$ & $\begin{array}{l}\text { antidepressive/ } \\
\text { antipsychotische Wirkung }\end{array}$ & 6 \\
\hline Nebenwirkung Methadon & & 3 \\
\hline sonstiges & & 1 \\
\hline
\end{tabular}


uns zur Weiterbehandlung übernommen. Drogenabhängig seit 1991. Hepatitis C seit 1998 bekannt.

\section{Weitere Diagnosen: Angststörung mit Panikattacken, Klaustrophobie, Depressionen, Schlafstörungen}

Insgesamt während der gesamten bisherigen Behandlungsdauer (und Teilnahme an den sonstigen Behandlungsmaßnahmen) ständiger Beikonsum von Opiaten. Dies „gehöre zu ihr", sie will zwar nichts mehr nehmen, doch der Drang ist dann stärker als ihr Wille („Craving“).

Versuch einer stationären Entgiftung und Langzeittherapie (Januar 2000) schlug fehl; Patientin brach die LZT nach einer Woche ab. Sie erschien wieder bei uns, um sich akupunktieren zu lassen und die ambulante Psychotherapie fortzusetzen. Rückfälligkeit wurde verneint. Dies widersprach unserem klinischen Eindruck, aber wegen der hohen Schamschwelle insistierten wir nicht weiter und hielten das Behandlungsund Beziehungsangebot aufrecht. Die Patientin entschloss sich im Februar zur erneuten medikamentösen Behandlung. Sie wünschte eine Nemexin-Behandlung als Rückfallprophylaxe, doch dazu „war es zu spät“. Eine Opiatfreiheit als Voraussetzung zur Nemexin-Behandlung ließ sich nicht erreichen. Die Rückfälligkeit wurde nicht mehr „verdrängt“, sondern thematisiert.

Wir entschieden uns, der Patientin Buprenorphin anzubieten, nicht trotz, sondern wegen des regelmäßigen Beikonsums, da wir hofften, dass vielleicht die anderen psychotropen Effekte von Buprenorphin den Konsum von Heroin „überflüssig“ machen würden. So wurde es auch mit der Patientin besprochen. Patientin konnte sich jedoch nicht entscheiden, da sie grundsätzlich Angst vor jeglicher Art von Tabletten habe.

Wir begannen am 24.2.00 mit $30 \mathrm{mg}$ Methadon; diese Dosis wurde beibehalten bis zum 5.3.00. Dann wünschte die Patientin eine Erhöhung der Dosis auf $40 \mathrm{mg}$ (6.3.00).

Am 7.3.00 wünschte sie doch eine Umstellung auf Buprenorphin. Sie erhoffte sich davon eine „Stabilisierung der Psyche“. Ungeschickterweise wurde die Methadondosis am Vortag noch auf $40 \mathrm{mg}$ erhöht.

Da unser erster „Subutex-Patient“ die Umstellung bei einer Methadonausgangsdosis von sogar $50 \mathrm{mg}$ problemlos vertrug, machten wir uns keine Sorgen.

Die zweite Umstellung lief nicht so glatt, der Patientin ging es sehr schlecht. Ob es an der zu hohen Methadondosis lag oder an der ängstlichen Struktur der Patientin, dazu können wir keine Aussage machen. Vermutlich haben sich beide Möglichkeiten verstärkt.

\section{Die Patientin litt unter folgenden Symptomen:}

Übelkeit, Angst, Schweißausbrüche, Gliederschmerzen, pelziges Gefühl. Wir behielten die Patientin fast den ganzen Tag in der Praxis und betreuten sie (Kaffee, Gespräch, Akupunktur, Entspannungsübungen). Kaum zu Hause, rief sie uns wieder an und schilderte aufgeregt und voller Angst ihren Zustand. Wir versuchten sie zu beruhigen, auch mit dem Hinweis, dass es Buprenorphin schon seit fast 30 Jahren gäbe und ihr sicher nichts Schlimmes passieren würde. Am Folgetag gab es ein langes Gespräch, ob weiter Buprenorphin oder wieder Methadon vergeben wird. Wir haben ihr gesagt, dass wir mit einer Rückumstellung keine Erfahrung haben und somit auch nicht versprechen können, dass es ihr dann sofort wieder besser gehe, wir aber aufgrund der Pharmakokinetik (Rezeptorbindung) eher davon ausgehen, dass es ihr so oder so einige Tage noch nicht gut gehen wird. Wir empfahlen eher ein rasches Aufdosieren. Die Patientin entschloss sich, „durchzuhalten“.

Sie erhielt in der ganzen ersten Woche besondere Aufmerksamkeit und Betreuung. Sie kam zur Ruhe, es ging ihr zunehmend besser. Nach einigen Tagen war sie froh und stolz auf sich, es durchgehalten zu haben.

\section{Dosierungsschema}

7.3.00: $6 \mathrm{mg}$, 8.3.00: $8 \mathrm{mg}, 19.3 .00$ bis jetzt: $12 \mathrm{mg} /$ die.

Ab dem 28.3.: alternierende Gabe von $24 \mathrm{mg}$. Wird physisch problemlos vertragen. Dennoch wünschte sich Patientin zwischenzeitlich (ab 3.4.00) wieder tägliche Gabe, da sie „den Kontakt" brauche. Seit dem 19.4.00 wieder zweitägliche Einnahme von $24 \mathrm{mg}$. Wir lassen dies die Patientin selbst bestimmen. Dass sie überhaupt inzwischen eigene Bedürfnisse anmelden kann, betrachten wir als Therapieerfolg.

\section{Weiterer Verlauf}

Der Patientin geht es gut. Sie gibt an, dass der Suchtdruck nachlasse. Auch sonst („im Kopf“) gehe es ihr besser. Sie gibt nach insgesamt 2 Jahren Behandlung die ersten opiatfreien Urinproben ab. Darauf ist sie sehr stolz.

Wir bemerken eine Stimmungsaufhellung und bessere Schwingungsfähigkeit, welche sich auch positiv auf die Psychotherapie auswirkt.

Diese Verbesserung des Verlaufes tritt trotz erheblicher familiärer und justizieller Probleme ein.

Auf unsere Frage, was ihrer Meinung nach zu der Verbesserung geführt habe (Wechsel des Medikamentes, Psychotherapie, Sonstiges), sagt sie: „Weiß ich nicht. Vielleicht alles zusammen.“

Die Struktur unserer Praxis erlaubt, verschiedene Behandlungsmethoden zu integrieren: Pharmakotherapie, Psychotherapie, Akupunktur, Autogenes Training. Bei der Patientin kommt die ganze Palette zur Anwendung.

\section{Schlussbemerkungen}

Wir sind der Ansicht, dass das „alleinige Auswechseln des Opiates" in den seltensten Fällen zur Behandlung der Opiatabhängigkeit ausreicht. Unabhängig von den nichtmedikamentösen Maßnahmen sollte jedoch, wie bei jeder anderen Krankheit, uns suchtmedizinisch tätigen Ärztinnen und Ärzten eine Palette von Medikamenten zur Verfügung stehen. Ein Schritt in diese Richtung ist durch die Zulassung von Buprenorphin erfolgt. Der Einsatz verschiedener Medikamente ist sinnvoll, da eine unterschiedliche Wirkung auf den Rezep- 
toren durchaus zu einer Verbesserung des Behandlungsverlaufes beitragen kann.

Verschiedene Studien [1] belegen, dass eine Behandlung der Depressivität bei Substituierten zu einer Verminderung des Opiatbeikonsums beiträgt. Dazu wird z.B. Doxepin verwendet. Unabhängig von bekannten Neben- und Wechselwirkungen sehen wir ein zusätzliches Problem bei der zweifelsohne oft indizierten Verordnung verschiedener Psychopharmaka. Da die abendliche Gabe nicht fremdkontrolliert erfolgt, wird die Dosis nicht nach Anweisung, sondern „nach Bedarf“ eingenommen. Häufig werden auch diese Medikamente verkauft.

Wir begrüßen, dass bei ohnehin stattfindender Opiatsubstitution das Substitut an sich durch seine spezifische Wirkung ( $\mu$-Agonismus, $\kappa$-Antagonismus) eine Begleitmedikation (als solche könnte auch Beikonsum interpretiert werden) überflüssig machen kann.

Auch erlaubt die Pharmakokinetik eine gewisse Flexibilität des Einnahmemodus, wobei auch auf Patientenwünsche eingegangen werden kann (einmal ausschlafen, freies Wochenende), ohne dass Regeln verletzt (und somit Grenzen überschritten) werden, was wiederum der Beziehungsarbeit dient.

Inzwischen ist auch die Verordnung von Buprenorphin im Rahmen der „Take-home“ Regelung möglich. Dadurch wir das Medikament für eine größere Patientengruppe attraktiv. Die frühere Restriktion ließ sich nicht durch die Pharmakologie der im Vergleich zu Methadon sicheren Substanz erklären. Das Ziel der „Take-home“-Verordnung stellt nicht zuletzt für zahlreiche Substituierte eine große Motivation dar, vom Beikonsum Abstand zu nehmen.

\section{Literatur}

${ }^{1}$ Paetzold W et al. Buprenorphin: Aspekte der Therapie von Opiatabhängigkeit, Depression und Schizophrenie. Nervenheilkunde 2000; 19: 143-150
Inge Hönekopp

Methadonschwerpunktpraxis Mannheim

L1, 1

68161 Mannheim

E-mail: KVMA_Methadon@t-online.de 